\title{
Intestinal epithelia activate anti-viral signaling via intracellular sensing of rotavirus structural components
}

\begin{abstract}
AH Frias ${ }^{1,2}$, M Vijay-Kumar ${ }^{1}$, JR Gentsch $^{3}$, SE Crawford ${ }^{4}$, FA Carvalho ${ }^{1}$, MK Estes $^{4}$ and AT Gewirtz $^{1,2}$
Rotavirus (RV), a leading cause of severe diarrhea, primarily infects intestinal epithelial cells (IECs) causing self-limiting illness. To better understand innate immunity to RV, we sought to define the extent to which IEC activation of antiviral responses required viral replication or could be recapitulated by inactivated $\mathrm{RV}$ or its components. Using model human intestinal epithelia, we observed that RV-induced activation of signaling events and gene expression typically associated with viral infection was largely mimicked by administration of ultraviolet (UV)-inactivated RV. Use of antiinterferon (IFN) neutralizing antibodies revealed that such replication-independent anti-viral gene expression required type I IFN signaling. In contrast, RV-induction of nuclear factor- $\kappa B-$ mediated interleukin-8 expression was dependent on viral replication. The anti-viral gene expression induced by UV-RV was not significantly recapitulated by RV RNA or $\mathrm{RV}$ virus-like particles although the latter could enter IEC. Together, these results suggest that RV proteins mediate viral entry into epithelial cells leading to intracellular detection of RV RNA that generates an anti-viral response.
\end{abstract}

\section{INTRODUCTION}

Rotavirus (RV) is the most common cause of severe dehydrating diarrheal disease in young children worldwide, causing up to 100 million cases and $>600,000$ deaths each year. ${ }^{1,2}$ RV infections are also common in adults, especially those who interact with children, but generally cause only mild symptoms. ${ }^{3} \mathrm{RV}$, a member of the Reoviridae family of viruses, is a non-enveloped double-stranded (ds) RNA virus containing three concentric protein layers. ${ }^{4,5}$ Its genome consists of 11 dsRNA segments that each code for different rotaviral proteins. Rotaviral proteins are divided into two groups, namely, structural proteins (VP 1-4, 6-7) that compose the viral structure and non-structural proteins (NSP1-6), which are synthesized during infection and function to facilitate viral replication or pathogenesis. ${ }^{4}$ VP4, a spike-shaped hemagglutinin protein that emanates through the outermost (VP7) protein layer, has a role in cellular attachment and must be cleaved by proteases normally present in the host intestine for $\mathrm{RV}$ to be infectious. ${ }^{4,5} \mathrm{NSP}$ proteins have a role in driving RVinduced diarrhea and suppressing host immune responses. ${ }^{4}$

$\mathrm{RV}$ infection is generally localized to the gastrointestinal tract and typically resolves within 7 days. ${ }^{1,6} \mathrm{RV}$ infection of mice lacking functional B and $\mathrm{T}$ lymphocytes often results in chronic infection, highlighting the importance of adaptive immunity in host defense to this pathogen. ${ }^{6,7}$ However, Franco and Greenberg ${ }^{7}$ observed that $40 \%$ of severe combined immunodeficient mice (on a C57BL/6 background) cleared RV infection. In addition, Eiden et al. ${ }^{8}$ observed clearance of RV in athymic Balb/c mice that was not accompanied by anti-RV antibodies. These findings suggest an important role for innate immunity in controlling RV infection. Such innate immune control of RV may be mediated by intestinal epithelial cells (IECs) because these cells are the predominant target of RV infection. The role of IEC in clearing RV infection is likely independent of RV-induced diarrhea in that the kinetics of clearance during primary infection are similar in newborn and adult mice although only the former show diarrhea. ${ }^{4}$ Numerous studies have noted that RV robustly induces nuclear factor (NF)- $\mathrm{KB}$-mediated epithelial expression of the chemokine interleukin (IL)- 8 , yet given that one of the hallmarks of RV infection is the absence of neutrophil infiltration, ${ }^{9-11}$ it is hard to envision IEC production of this chemokine has a major role in clearance of RV. Rather, based

${ }^{1}$ Department of Pathology, Emory University, Atlanta, Georgia, USA. ${ }^{2}$ Immunology and Molecular Pathogenesis Graduate Program, Emory University, Atlanta, Georgia, USA. ${ }^{3}$ Division of Viral Diseases, Viral Gastroenteritis Section, Centers for Disease Control, Atlanta, Georgia, USA. ${ }^{4}$ Department of Molecular Virology and Microbiology, Baylor College of Medicine, Houston, Texax, USA. Correspondence: AT Gewirtz (agewirt@emory.edu)

Received 10 March 2010; accepted 14 June 2010; published online 21 July 2010. doi:10.1038/mi.2010.39 
on the large body of data on host responses to viral infections in general, it seems more plausible that type 1 interferon (IFN) responses contribute to innate immune-mediated clearance of RV. In accordance, gene-profiling studies of RV-infected IEC observed elevated expression of a panel of genes related to type I IFN responses. ${ }^{12}$ Pretreatment of cultured cells with type 1 IFNs limited RV infection. ${ }^{13}$ Levels of type I IFNs increase in RV-infected children and animals and administration of exogenous type I IFN reduces disease sequellae in cattle and pigs. ${ }^{4,14-16}$ Moreover, RV uses the non-structural protein NSP1 to suppress IFN signaling. ${ }^{17,18}$ Mutations in NSP1 that ablate RV's ability to interfere with IFN-related signaling attenuate RV's spread to uninfected cells, ${ }^{17}$ further supporting the notion that IFN signaling is a potential hindrance to this pathogen. Although mice deficient in type I and II IFN receptors are able to clear $\mathrm{RV},{ }^{19}$ loss of the transcription factor signal transducer and activator of transcription 1 (STAT1), which mediates much of the gene expression induced by type I IFN, severely impairs control of $\mathrm{RV}^{20}$ Thus, although there is considerable redundancy in host defense mechanisms against RV, it seems likely that IEC activation of STAT1 and induction of genes regulated by type I IFN have an important role in host defense against this virus. Therefore, we sought to analyze the mechanism by which IEC activate such anti-viral signaling events in response to RV infection.

Viral infection has long been known to alter host gene expression by inducing endoplasmic reticulum (ER) stress, sometimes called "ER overflow", in which the rapid switch in translation from host-encoded to viral-encoded proteins activates host stress-related transcription factors. ${ }^{21}$ This model predicts that an ultraviolet (UV)-inactivated virus, which cannot replicate or express its genes, would not alter host gene expression. In accordance, inactivation of the RV genome through UV-irradiation markedly reduces RV's ability to induce NF- $\mathrm{KB}$ activation and IL-8 secretion. ${ }^{10,11}$ More recently, it has become appreciated that a large portion of microbe-induced gene expression does not require a viable microbe per se but, rather, results from host pattern-recognition receptors (PRRs) such as the toll-like receptors detecting various microbial components. For example, IEC detection of motile bacteria is largely mediated by toll-like receptor-5 detection of bacterial flagellin. ${ }^{22}$ However, IEC seem to be hyporesponsive to a number of other toll-like receptor agonists, highlighting the stark differences in the mechanism by which different microbes might be detected by IEC. ${ }^{23}$ IEC have been observed to respond to synthetic dsRNA, poly (I:C), resulting in expression of both IL-8 and a panel of genes associated with type I IFN responses, suggesting IEC have the potential to respond to RV through detection of viral RNA. ${ }^{24}$ However, whether such observations could apply to an actual virus-IEC interaction remained unclear. Thus, the goal of this study was to define the extent to which IEC response to RV results from viral replication or, rather is largely a consequence of IEC detection of RV components. We observed that RV-induced IEC antiviral signaling was almost entirely driven by detection of viral components, likely resulting from VP4-mediated viral entry followed by detection of RV RNA.

\section{RESULTS \\ Apical infection by RV induces epithelial anti-viral signaling}

Epithelial cell-mediated innate immune responses have a potentially important role in protecting the host against RV. Yet, although some studies of RV-induced innate immune activation have focused on activation of NF- $\kappa \mathrm{B}$-mediated genes such as IL-8, RV-induced anti-viral signaling in IECs have not been extensively studied. Thus, we infected human HT-29 IECs with RV (multiplicity of infection (MOI) 0.5-1) and temporally assayed signaling events typically associated with a variety of viral infections. Mock-infected control samples were exposed to low levels of trypsin, which may activate protease-activated receptors, however, such treatment was included because it is required for cleavage of RV capsid protein VP4 and thus allows for robust infectivity. ${ }^{25} \mathrm{RV}$ infection induced transient phosphorylation of transcription factors interferon regulatory factor (IRF3) and STAT1 with a maximal response being observed between 8 and 16 h postinoculation (h.p.i) (Figure 1a). RV also induced epithelial secretion of IFN- $\beta$ (Figure 1b) and, in accordance with other studies, IL-8 (Figure 1c). Interestingly, RV-induced IL-8 production was relatively delayed suggesting it may not reflect immediate IEC sensing of the virus. These signaling events, which were not observed in mock-infected cells, roughly correlated with levels of the viral protein VP6 and are consistent with the finding that viral replication is required for $\mathrm{RV}$-induced NF- $\mathrm{\kappa B}$ activation. ${ }^{11}$ We next performed these experiments in polarized IEC, which result when IEC are cultured on collagen-coated permeable supports, in the hope that doing so might provide insight into mechanisms underlying such RV-induced anti-viral signaling. For example, use of polarized IEC previously enabled us to uncouple Salmonella invasion from activation of innate immunity, ultimately allowing definition of mechanisms underlying the latter process. ${ }^{26}$ Consistent with previous findings, ${ }^{27} \mathrm{RV}$ infection was markedly more efficient when the virus was administered to the apical surface of epithelia (Figure 1d). Such reduced infectivity of basolaterally administered RV correlated with marked attenuation of IRF7, STAT1, and protein kinase R (PKR) activation and induced secretion of IFN- $\beta$ and IL- 8 (Figures 1d-f). Such preferential infection and induction of anti-viral signaling by apical RV argues against RV-activating innate immune signaling through a basolateral PRR, as occurs in response to Salmonella, ${ }^{28}$ but seems consistent with the possibilities that anti-viral signaling might be triggered by an apical receptor, an intracellular receptor, or result from viral replication causing ER stress.

\section{Inactivated RV induces anti-viral gene expression similar to live RV}

To determine the extent to which RV-induced anti-viral signaling required viral replication or could be mimicked by structural components of RV, we examined the epithelial response to UVirradiated RV (UV-RV), which is structurally intact but rendered non-replicative. ${ }^{29,30}$ The inability of UV-RV to replicate in IEC was verified by monitoring levels of VP6 over time (Figure 2a). In accordance with previous studies, such UV-inactivation of the 
a

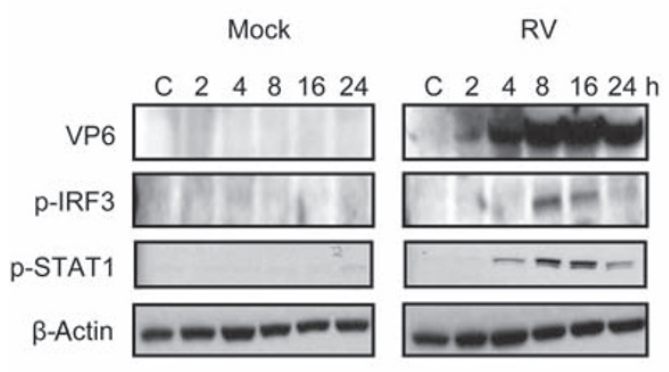

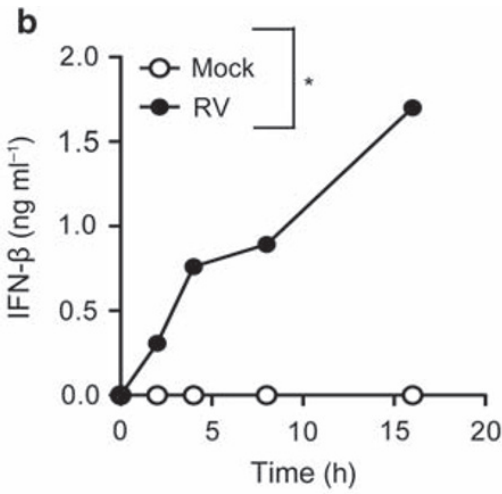

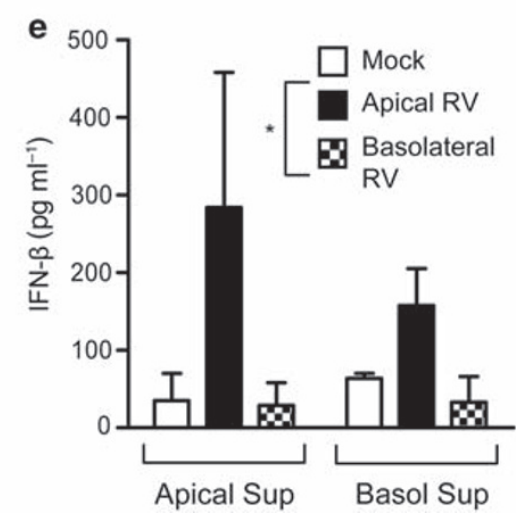

C
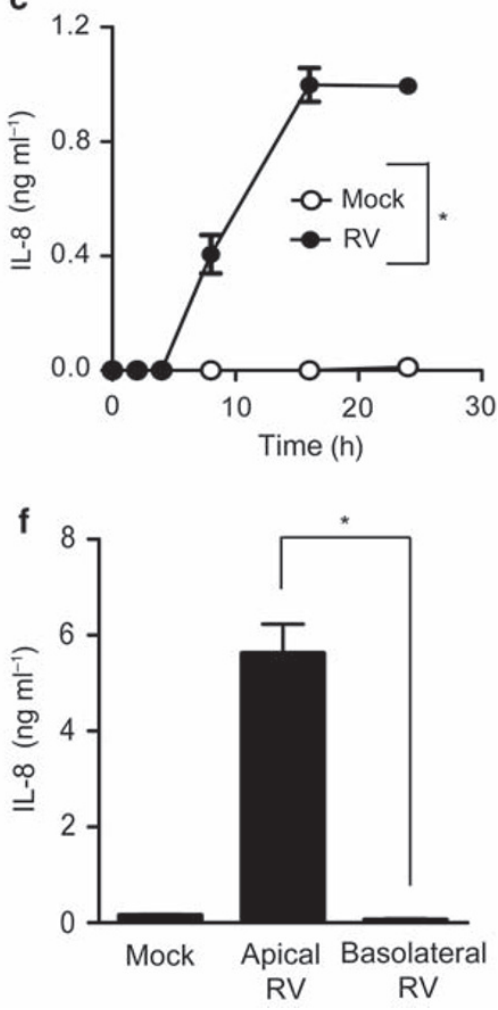

d

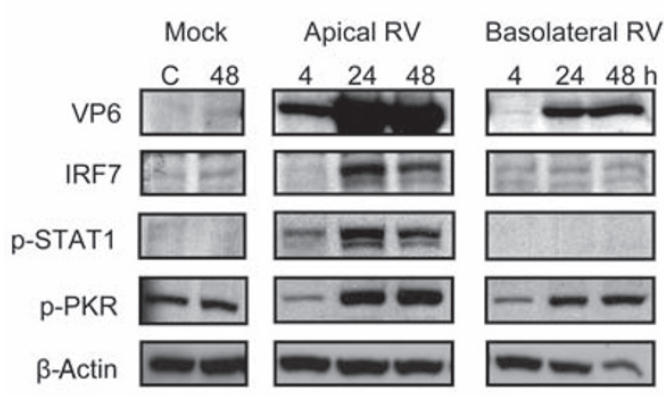

Figure 1 Anti-viral protein expression in rotavirus (RV)-infected intestinal epithelia. Human intestinal epithelia (HT29) were grown to confluence in six-well plates $(\mathbf{a}-\mathbf{c})$ or collagen-coated permeable supports $(\mathbf{d}-\mathbf{f})$ and infected with RV (MOI 0.5-1). Control samples were treated with trypsin diluted in serum-free medium (SFM) (mock) or SFM alone (c). Cell lysates and supernatants were collected 0-48h post-inoculation (h.p.i.). Western blot analyses were performed to assess viral protein (VP6) synthesis and protein expression of the indicated anti-viral markers in cell lysates (a, d). Enzyme-linked immunosorbent assay (ELISA) assays were used to measure interferon (IFN)- $\beta$ (b, e) and interleukin (IL)-8 (c, f) secretion in supernatants over time. Data in $(\mathbf{a}, \mathbf{b}, \mathbf{d}$, and $\mathbf{e})$ are results of a single experiment and representative of three separate experiments that gave similar results. Data in (c and $\mathbf{f}$ ) reflect the mean \pm standard error of the mean (s.e.m.) of three parallel experiments. Statistically significant differences $P<0.05$ are denoted as starred values $\left(^{*}\right)$.

RV genome substantially reduced induction of IL-8 (Figure 2c). However, in contrast, signaling events typically associated with viral infection including activation of STAT1 and IRF3/7 and induction of IFN- $\beta$ secretion, were elicited at least as robustly by UV-RV (Figures $2 \mathbf{a}$ and $\mathbf{b}$ ). Similar activation of anti-viral signaling by RV and UV-RV was also observed in polarized epithelia in response to apical stimulation (data not shown). To confirm these events were indeed induced by UV-RV as opposed to UV cross-linked cell debris that might have been present in our virus preparation, we performed a control experiment showing that UV-irradiation of a mock viral preparation, which contained MA104 cell debris but not RV, did not elicit anti-viral signaling induced by UV-RV (Figure 2d). Next, we determined if trypsinization, which is known to be required for viral entry, is also required for anti-viral signaling in response to UV-RV. Indeed, robust activation of anti-viral signaling by both RV and UV-RV required the stimulating agonist to be treated with trypsin before IEC stimulation (Figure 3). Finally, we observed that, analogous to the case for RV, activation of innate immune signaling in response to UV-RV was more robust when UV$\mathrm{RV}$ was applied to the apical rather than basolateral surface of polarized epithelia (Figure 4). Together, these results suggest that UV-RV induces anti-viral signaling through a mechanism similar to live virus, and further supports the notion that type I IFN is activated by IEC detection of RV structural components rather than viral replication.

We next sought to define the extent to which RV-induced gene expression in general can be mimicked by UV-RV and determine the role of type I IFN in RV-induced changes in IEC gene expression. IEC were mock-infected or exposed to RV, UV$\mathrm{RV}$, or RV in the presence of neutralizing antibodies to type 1 IFN (anti-IFN $\alpha / \beta)$ for $24 \mathrm{~h}$, at which time gene expression was assayed by complementary DNA microarray. The gene chip used for this purpose permits simultaneous examination of 12 different samples allowing us to assay 4 different experimental conditions in biological triplicates, thus permitting statistical analysis to be performed directly on the microarray data. Such microarray analyses indicated that RV upregulated 1,190 genes by at least 1.3-fold relative to mock-treated uninfected cells (cut-off was arbitrarily chosen based on our previous experience with microarray-based studies of IEC $^{24}$ ). The entire microarray data set is available on-line (posted on gene expression omnibus) and some of the common means of examining microarray data such as unsupervised clustering analysis are shown in Supplementary data online (Supplementary Figure 1 online). In Figure 5a, we sought to show our microarray data in a manner that would most facilitate 


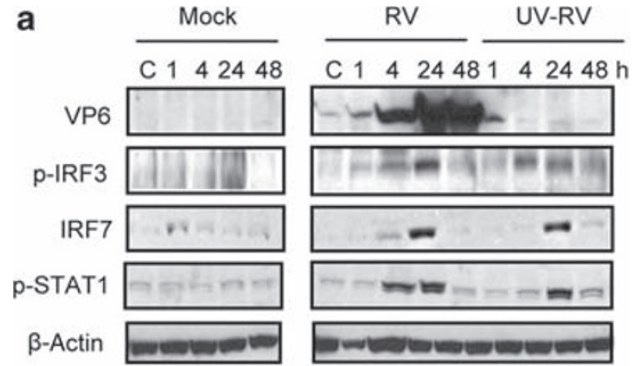

b

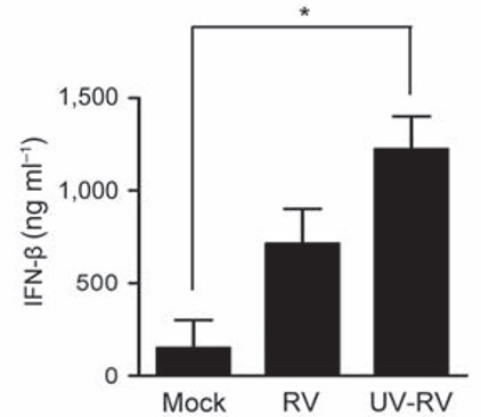

C

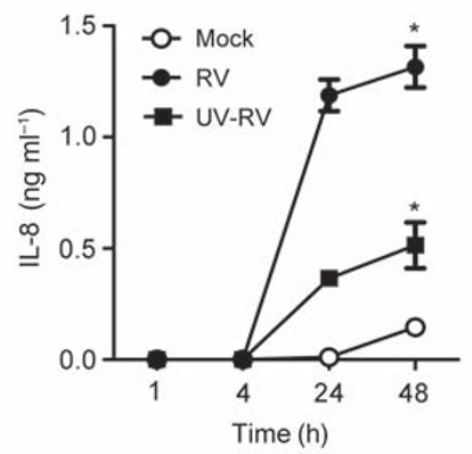

d

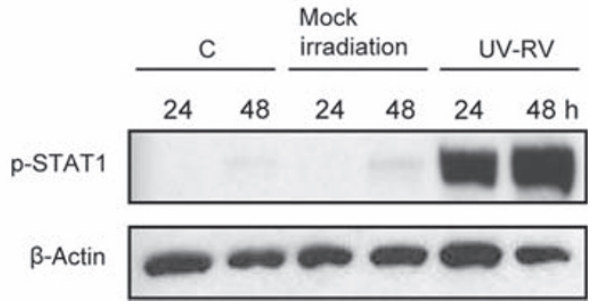

Figure 2 Anti-viral protein expression showed in rotavirus (RV)infected and ultraviolet (UV)-RV stimulated epithelia. Confluent intestinal epithelia (HT29) were grown in six-well plates and treated with RV and UV-RV (MOI 1). Control samples were exposed to trypsin diluted in serum-free medium (SFM) (mock), irradiated cellular debris from a mock preparation of UV-RV (mock irradiation), or SFM alone (c). Cell lysates and supernatants were collected at various time points (0-48h.p.i.). Western blot analyses were performed to assess viral protein (VP6) synthesis and protein expression of the indicated anti-viral markers in cell lysates (a, d). Enzyme-linked immunosorbent assay (ELISA) assays were used to measure secretion of interferon (IFN)- $\beta$ (b) and interleukin (IL)-8 (c) in supernatants at 48 h.p.i. Data in (a, b, and $\mathbf{d})$ are results of a single experiment and representative of three separate experiments that gave similar results. Data in (c) are the mean \pm s.e.m. of three parallel experiments. Statistically significant differences $P<0.05$ are denoted as starred values $\left({ }^{*}\right)$.

addressing our central questions. Specifically, we generated a "heat map" that shows gene expression in each of the 12 samples ( 4 conditions, 3 replicates) relative to the average expression of mock-treated cells. The relative uniformity of the three replicates in each condition indicates the high degree of similarity among our biological replicates. Genes were ordered (top to bottom) based on their relative dependence on type I IFN (ratio of expression on exposure to RV alone vs. RV in the presence of anti-IFN $\alpha / \beta$ ). A majority of genes that were induced by RV are thus type I IFN dependent in that their expression was reduced by the neutralizing antibody. The heat map shows that the vast majority of such IFN-dependent gene expression did not seem to require viral replication in that almost of all of these genes were similarly induced by both RV and UV-RV. Such type I IFNdependent, replication-independent, RV-induced gene expression included a panel of classic anti-viral genes such as IRF7, IFN- $\beta$, STAT1, Mx1, oligoadenylate synthetase- 2 , and major histocompatibility complex I (Table 1). Use of quantitative reverse transcriptase-PCR (qRT-PCR) verified the upregulated mRNA levels of some of these genes (Figures $\mathbf{5 b}-\mathbf{e}$ ). In contrast to the induction of such classic anti-viral genes, expression of IL-8 was partially dependent on viral replication (Figure 2c) and independent of type I IFN (Table 1). Thus, a large portion of $\mathrm{RV}$-induced gene expression in IEC, particularly upregulation of genes typically associated with viral infection, is independent of viral replication and dependent on type I IFN.

\section{$\mathrm{RV}$ components fail to recapitulate $\mathrm{RV}$-induced IEC anti-viral signaling}

Next, we sought to better define the structural determinant of RV that had an important role in activating IEC anti-viral signaling. Specifically, we compared epithelial responses with UV-RV, RV virus-like particles (VLPs), and purified RV RNA. The VLPs used here are protein shells comprised of four major RV structural proteins (VP 2/4/6/7) that lack nucleic acid. ${ }^{31}$ RV VLPs were used at protein concentrations of $0.5-5 \mu \mathrm{g} \mathrm{m}^{-1}$, which is equivalent to the concentration of UV-RV that corresponds to MOIs of 1-10. Purified RV RNA was used at concentrations of $0.5-5 \mu \mathrm{g} \mathrm{ml}^{-1}$, which is approximately 100 times the amount of RNA in UV-RV that corresponds to MOIs of 1-10. In contrast to UV-RV, neither RV VLPs nor RNA induced detectable elevations in levels of phospho-STAT1 or IRF7 (Figure 6a). RV VLPs and RNA also failed to recapitulate the induction of IFN- $\beta$ or IL- 8 elicited by UV-RV (Figures $\mathbf{6 b}$ and c). To more broadly understand the extent to which these components of UV-RV might recapitulate its ability to activate gene expression in epithelial cells, we measured changes in epithelial gene expression through microarray analysis. A modest concentration of UV-RV (MOI 0.5) induced 401 genes by $>1.3$-fold relative to mock-treated control cells including key anti-viral genes such as melanoma differentiation-associated gene 5 , IFN- $\beta$, and major histocompatibility complex I (Tables 2 and 3). Only a small portion of the genes upregulated by UVRV were similarly induced by treatment with RV VLPs or RNA (Table 2). In accordance, induction of anti-viral gene expression by RV viral components was also not observed to be comparable to UV-RV (Table 3), and these trends were confirmed by measuring mRNA synthesis of select anti-viral genes through qRT-PCR (Figures 6d and e). Thus, neither RV RNA 
a

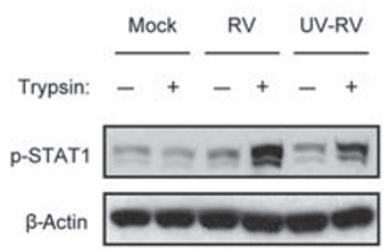

b

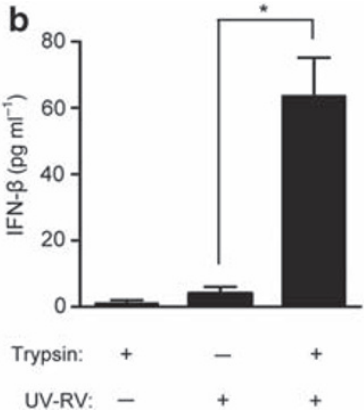

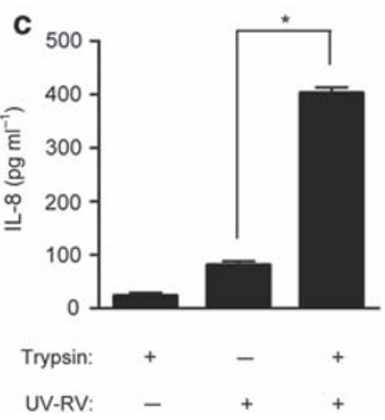

Figure 3 Anti-viral protein expression showed in epithelia treated with rotavirus (RV) and ultraviolet (UV)-RV in the presence or absence of trypsin. Intestinal epithelial monolayers (HT29) grown in six-well plates were treated with RV and UV-RV (MOI 1) in the presence or absence of trypsin (24h.p.i.). Control samples received equivalent amounts of trypsin diluted in serum-free medium (SFM) (mock) or SFM alone. Trypsin and trypsin-free treatments are denoted as $(+)$ and $(-)$ symbols, respectively. Western blot analysis was used to detect anti-viral gene expression in cell lysates (a). Enzyme-linked immunosorbent assay (ELISA) assays were performed to measure secretion of interferon (IFN)- $\beta$ (b) and interleukin (IL)-8 (c) in supernatants at 24 h.p.i. Data in (a) show results of a single experiment and are representative of three separate experiments that gave similar results. Data in (b and $\mathbf{c})$ are the mean \pm s.e.m. of three parallel experiments. Statistically significant differences $P<0.05$ are denoted as starred values $\left({ }^{*}\right)$.

a

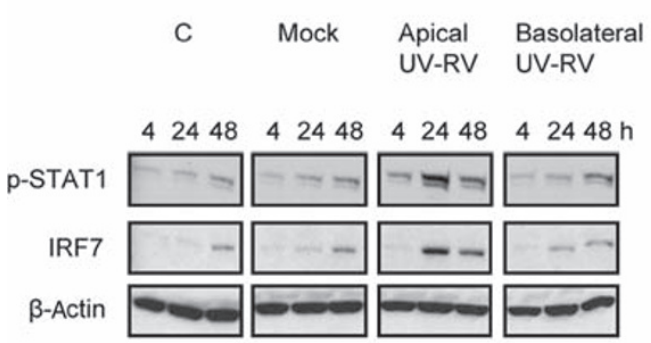

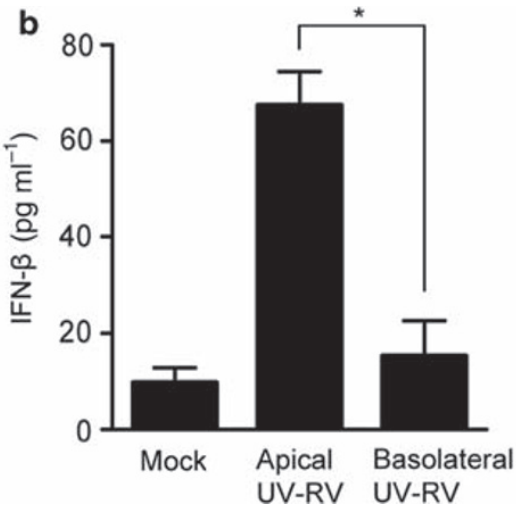

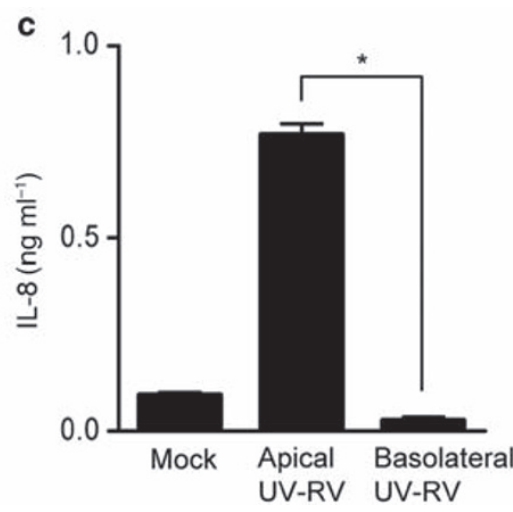

Figure 4 Anti-viral protein expression showed in epithelia treated apically and basolaterally with ultraviolet (UV)-rotavirus (RV). Intestinal epithelial monolayers (HT29) were grown on collagen-coated permeable supports and infected either apically or basolaterally with UV-RV (MOI 1). Control samples received equivalent amounts of trypsin diluted in serum-free medium (SFM) (mock) or SFM alone (c). Cell lysates and supernatants were collected at various time points (0-48h.p.i.). Western blot analyses were performed to assess protein expression of the indicated anti-viral markers in cell lysates (a). Enzyme-linked immunosorbent assay (ELISA) assays were used to measure interferon (IFN)- $\beta$ (b) and interleukin (IL)-8 (c) secretion in supernatants at 48 h.p.i. Data in (a) show results of a single experiment and are representative of three separate experiments that gave similar results. Data in ( $\mathbf{b}$ and $\mathbf{c}$ ) reflect the mean \pm s.e.m. of three parallel experiments. Statistically significant differences $P<0.05$ are denoted as starred values $\left({ }^{*}\right)$.

nor VLPs could substantially recapitulate the changes in gene expression induced by inactive but structurally intact RV.

\section{RV and UV-RV show similar cell entry kinetics}

To better understand the interaction of UV-RV and RV VLPs with polarized epithelia and activation of epithelial anti-viral signaling in response to $\mathrm{RV}$, we examined the interaction of $\mathrm{RV}$, UV-RV, and RV VLPs with model epithelia through confocal microscopy for up to 4 h.p.i. (Figures $7 \mathbf{a}$ and $\mathbf{b}$ ). RV and UV-RV (MOI 10), and RV VLPs roughly equivalent to the estimated protein concentration of the RV preparation, were applied to the apical surface of epithelia for 1 and 4 h.p.i., followed by washing off of non-adsorbed or non-adhered materials. As expected, planar images of IEC taken $3 \mu \mathrm{m}$ below the apical surface revealed the presence of RV, UV-RV, and RV VLPs in the sub-apical region within 1 h.p.i. (Figure 7a). RV increased in abundance by 4 h.p.i., reflecting viral replication, while UV-RV levels decreased likely because of degradation of viral proteins and an inability to replicate (Figure $7 \mathbf{b}$ ). Thus, consistent with models by which RV is internalized by IEC, RV's primary structural proteins are sufficient to mediate its entry into IEC. Taken together, we interpret our results to suggest that RV activation of anti-viral signaling requires viral structural proteins to mediate entry of viral RNA into epithelial cells in which it can be detected by host PRR(s).

\section{DISCUSSION}

The ability of adult humans and mice to efficiently clear RV without major sequellae serves as an example of effective mucosal immunity. Thus, understanding host immunity to $\mathrm{RV}$ may provide insights into understanding the pathogenesis of viruses that are not dispatched in such an expedient manner. Such adept handling of RV likely reflects considerable redundancy in the mechanisms that protect the host against $\mathrm{RV}$. In accordance, and in contrast to the case for many viruses, mice lacking either adaptive immunity or type I IFN still show substantial control of RV infection. ${ }^{7,19}$ Although not required for clearance, type I IFNs modulate the course 
a
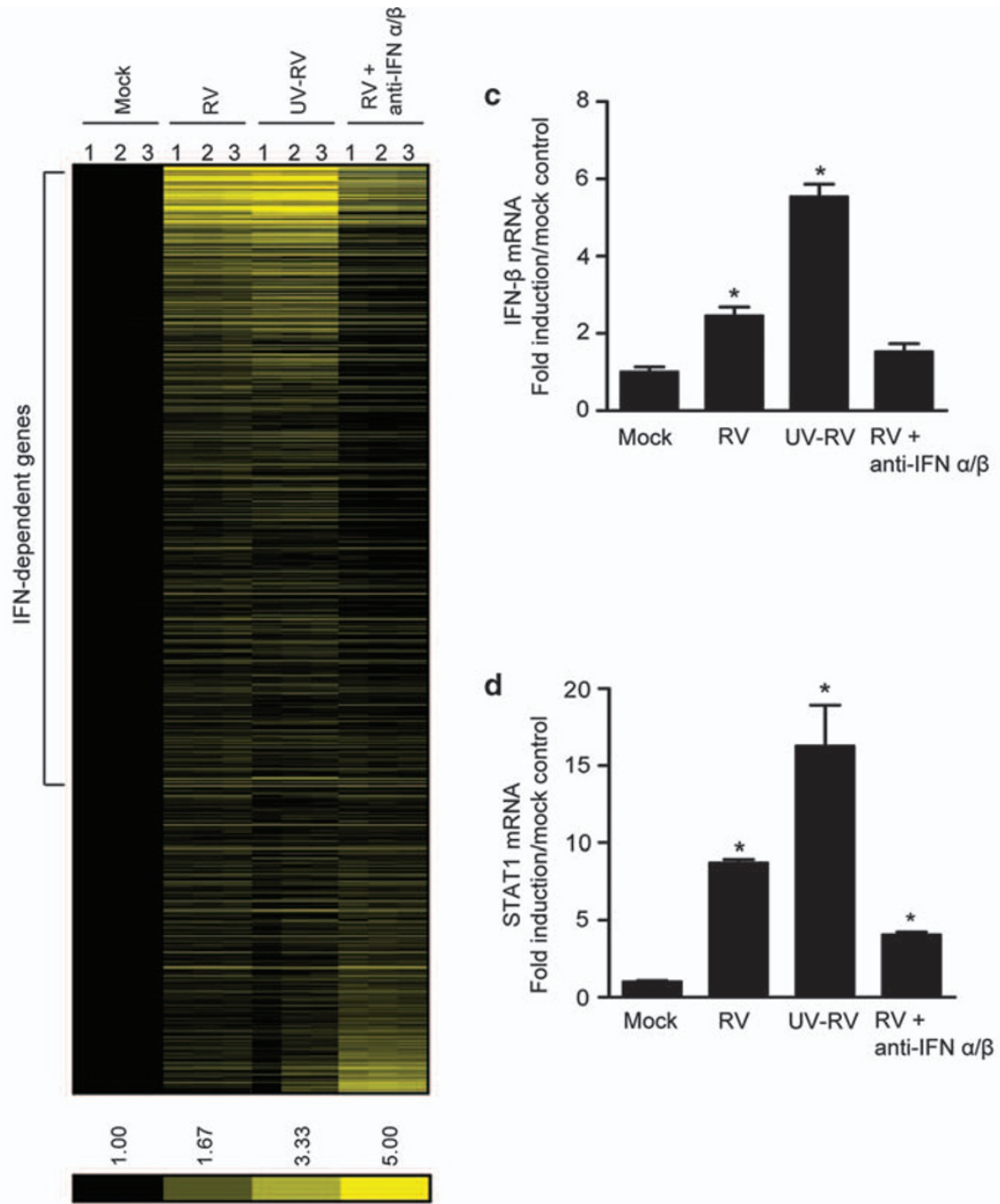

b
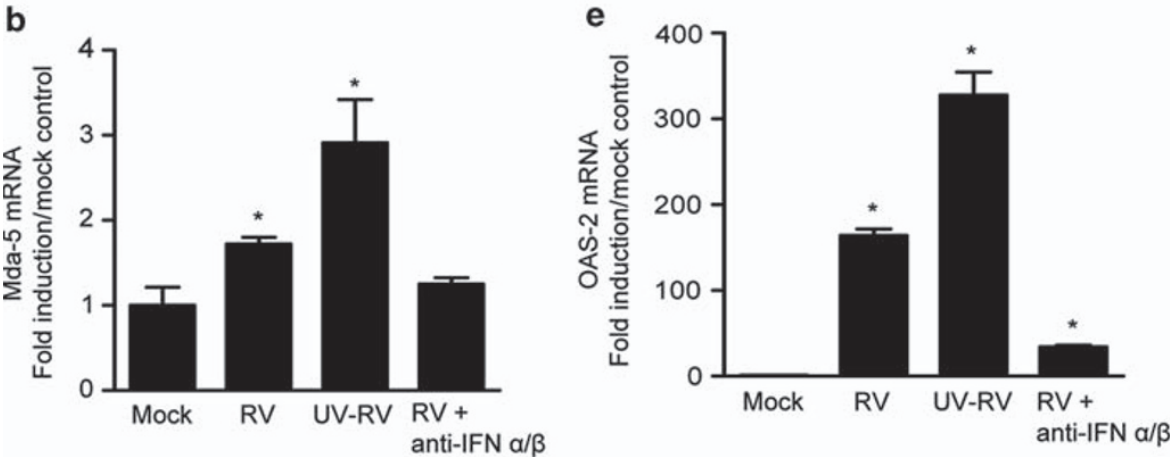

Figure 5 Transcription profiles of epithelia stimulated with rotavirus (RV), ultraviolet (UV)-RV, and RV in the presence of type 1 interferon (IFN) ( $\alpha / \beta)$ antibodies. Intestinal epithelial (HT29) cell monolayers were grown in six-well plates and infected with RV and UV-RV (MOI 1), and RV (MOI 1) plus type 1 IFN antibodies (anti-IFN $\alpha / \beta$ ). Control samples received equivalent amounts of trypsin diluted in serum-free medium (SFM) (mock). Experiments were performed in biological triplicates. At 24 h.p.i., cell lysates were extracted for RNA and microarray analyses were performed to assess global transcription of genes. Heat map illustration of genes induced by RV and UV-RV with $>1$.3-fold change relative to mock (a). Quantitative reverse transcriptase-PCR (qRT-PCR) results used to confirm mRNA synthesis of select anti-viral genes (b-e). Data in (a) show results of three parallel experiments. Data in (b-e) reflect the mean \pm s.e.m. of three parallel experiments. Statistically significant differences $P<0.05$ are denoted as starred values $\left(^{*}\right)$.

of RV infection in a variety of experimental systems $s^{4,13-15}$ and deletion of STAT1, a transducer of IFN-related signals, severely impairs control of RV. ${ }^{20}$ Thus, activation of STAT1/ type I IFN signaling is likely one important means of host defense against RV. Therefore, the goal of this study was to investigate how the predominant cell type infected by RV, the IEC, activates such anti-viral signals in response to RV infection. 
Table 1 Fold change induction of anti-viral markers relative to mock

\begin{tabular}{lccc}
\hline & RV & UV-RV & $\begin{array}{c}\text { RV }+ \text { anti-IFN } \\
\text { al } \beta\end{array}$ \\
\hline TLR3 & $1.5 \pm 0.07$ & $2.2 \pm 0.37$ & $1.13 \pm 0.09$ \\
Mda-5 & $8.7 \pm 1.2$ & $11.1 \pm 0.84$ & $4.24 \pm 0.71$ \\
RIG-I & $3.7 \pm 0.33$ & $6.6 \pm 0.39$ & $2.41 \pm 0.29$ \\
IRF3 & $1.3 \pm 0.06$ & $1.3 \pm 0.06$ & $1.16 \pm 0.07$ \\
IRF7 & $5.9 \pm 0.24$ & $7.7 \pm 0.90$ & $3.92 \pm 0.20$ \\
IFN- $\alpha$ & $1.1 \pm 0.06$ & $1.5 \pm 0.04$ & $0.95 \pm 0.08$ \\
IFN- $\beta$ & $11 \pm 0.88$ & $31.6 \pm 4.4$ & $6.24 \pm 0.84$ \\
STAT1 & $10.5 \pm 1.1$ & $12.8 \pm 0.85$ & $4.42 \pm 0.28$ \\
MX1 & $35.6 \pm 0.44$ & $45.2 \pm 3.9$ & $12.3 \pm 0.37$ \\
PKR & $3.3 \pm 0.18$ & $3.3 \pm 0.22$ & $1.72 \pm 0.18$ \\
OAS-2 & $43.6 \pm 2.3$ & $76.5 \pm 4.2$ & $11.6 \pm 1.2$ \\
MHC I & $11.6 \pm 0.92$ & $24.1 \pm 1.7$ & $4.1 \pm 0.64$ \\
IL-8 & $60 \pm 5.9$ & $24 \pm 2.2$ & $58 \pm 5.3$ \\
IFITM1 & $44.4 \pm 1.9$ & $112.6 \pm 4.9$ & $8 \pm 0.92$ \\
IFITM3 & $28.9 \pm 2.3$ & $37.3 \pm 2.9$ & $6.4 \pm 0.83$ \\
CXCL10 & $35.5 \pm 3.7$ & $108 \pm 11.3$ & $17.6 \pm 2.3$ \\
ISG15 & $10.4 \pm 3.0$ & $15.4 \pm 3.3$ & $5.03 \pm 0.90$ \\
\hline Ab & & & \\
\hline
\end{tabular}

Abbreviations: CXCL, CXC chemokine ligand; IFITM, interferon-induced transmembrane protein; IFN, interferon; IL, interleukin; ISG, interferon-stimulated gene; Mda-5, melanoma differentiation-associated gene 5; MHC, major histocompatibility complex; OAS, oligoadenylate synthetase; PKR, protein kinase R; qRT-PCR, quantitative reverse transcriptase-PCR; RIG, retinoic acid inducible gene 1; $\mathrm{RV}$, rotavirus; TLR, Toll-like receptor; UV, ultraviolet.

Anti-viral gene expression in cells treated with RV, UV-RV, and RV + anti-IFN $\alpha / \beta$ Intestinal epithelial (HT29) cell monolayers were stimulated with RV and UV-RV (MOI 1), RV (MOI 1) plus type 1 IFN antibodies (anti-IFN $\alpha$, anti-IFN $\beta$ ), and mock treatments as described in Figure 5. At 24 h.p.i., lysates were collected in TRIzol to assess global transcription of genes (Figure 5a), including antiviral markers of interest as indicated above. mRNA expression of select anti-viral genes was confirmed via qRT-PCR analysis (Figures $5 \mathbf{b}-\mathbf{e}$ ). Fold induction values greater than s.d. calculations reflect statistically significant differences from mock $(P<0.05)$.

We observed that, in contrast to RV activation of NF- $\kappa \mathrm{B}$, which is driven by synthesis of viral proteins that may cause ER stress, ${ }^{11} \mathrm{RV}$-induced anti-viral signaling was almost entirely driven by IEC detection of viral components. Indeed, while adding RV and inactivated RV (UV-RV) at the same MOI initially delivered similar levels of viral components, UV-RV antigens were degraded within a few hours while RV replication resulted in increasing levels of viral proteins over the ensuing $24 \mathrm{~h}$. Yet, a greater level of anti-viral signaling was observed in response to UV-RV, largely reflecting higher induction of type I IFN. That such higher levels of viral proteins in RV-infected cells did not result in higher IFN levels than was observed in cells exposed to UV-RV likely reflects the ability of RV to suppress IFN signaling/ expression through NSP1-mediated degradation of IRF 3, 5, and 7. ${ }^{17,18}$ Such ability of RV to suppress what might otherwise be a very robust anti-viral signal in response to the presence of even modest amounts of viral components speaks to the potential of these innate immune signals to limit RV infection.

Although a large portion of RV-induced IEC gene expression was dependent on type I IFN, including numerous genes typi- cally associated with viral infection, RV nonetheless induced numerous genes whose induction did not require type I IFN. Such RV-induced gene expression was not substantially recapitulated by UV-RV suggesting it results from ER stress and/or requires higher levels of viral components than was achieved by treatment with UV-RV. RV replication-dependent induced genes includes several NF- $\kappa B$-regulated pro-inflammatory genes including the neutrophil chemoattractant IL-8, which was one of the genes most highly induced by RV. That RV infection is not typically associated with cellular inflammation makes the role of such chemokine induction somewhat enigmatic. Yet, given that purified bacterial flagellin, which primarily activates NF- $\kappa \mathrm{B}-$ mediated pro-inflammatory gene expression provided mice with temporary protection against $\mathrm{RV}$ infection, ${ }^{32}$ we speculate that ER stress-mediated NF- $\kappa B$ activation may also be a redundant mechanism by which IEC limit RV infection.

The potent induction of anti-viral signaling observed in response to UV-RV was not mimicked by RV VLPs or RV RNA. The failure of RV VLPs to activate anti-viral signaling could conceivably reflect that RV non-structural proteins, which are not present in VLPs, have a role in activation of innate immunity. However, it seems more likely that IEC detection of RV RNA is required to generate robust anti-viral signaling. The failure of RV RNA to robustly activate anti-viral signaling likely resulted from lack of significant uptake by IEC. In accordance, IEC uptake and responses to synthetic dsRNA, poly(I:C), required that poly(I:C) be administered at high concentrations and correlated with its uptake. ${ }^{24}$ In vivo, and perhaps in vitro, free RNA that is not rapidly taken up by IEC would likely be quickly degraded by RNAses. In contrast, surface expression of VP4 on RV, UV-RV, and RV VLPs results in efficient internalization on encountering the sialic acid residues abundant on the apical surface of gut epithelia, ${ }^{27}$ enabling intracellular detection of RV dsRNA. The identity of such sialylated proteins, and the question of whether they engage other proteins during RV internalization, remains elusive. Highlighting the difference in the way that free dsRNA would interact with epithelia, we note that in contrast to RV and UV-RV, which preferentially enter IEC and activate anti-viral signaling when applied to the apical surface, uptake of poly(I:C) and subsequent signaling was much greater when administered to the basolateral surface. ${ }^{24} \mathrm{After} \mathrm{RV}$ entry, its RNA could be recognized by endosomal PRRs such as toll-like receptor-3 or by cytosolic detectors of dsRNA such as retinoic acid inducible gene 1 and melanoma differentiation-associated gene 5 . $\mathrm{PKR}$ is known to have a role in RV-induced gene expression but whether PKR is a true dsRNA receptor or is purely a participant in the dsRNA-activated signaling cascade remains unclear.

In conclusion, inactivation of the RV genome does not ablate but rather augments its induction of anti-viral signaling in IEC. These findings parallel observations made by us and others that treatment of epithelial cells with heat-killed bacteria and/or purified bacterial components induce greater activation of innate immune signaling than live bacteria. Such a finding supports the general paradigm that innate immune responses are largely driven by host PRR and do not require viable microbes per se. Rather, antimicrobial responses occur in spite of the complex mechanisms used by microbes to suppress them. Consequently, inactivated microbes 


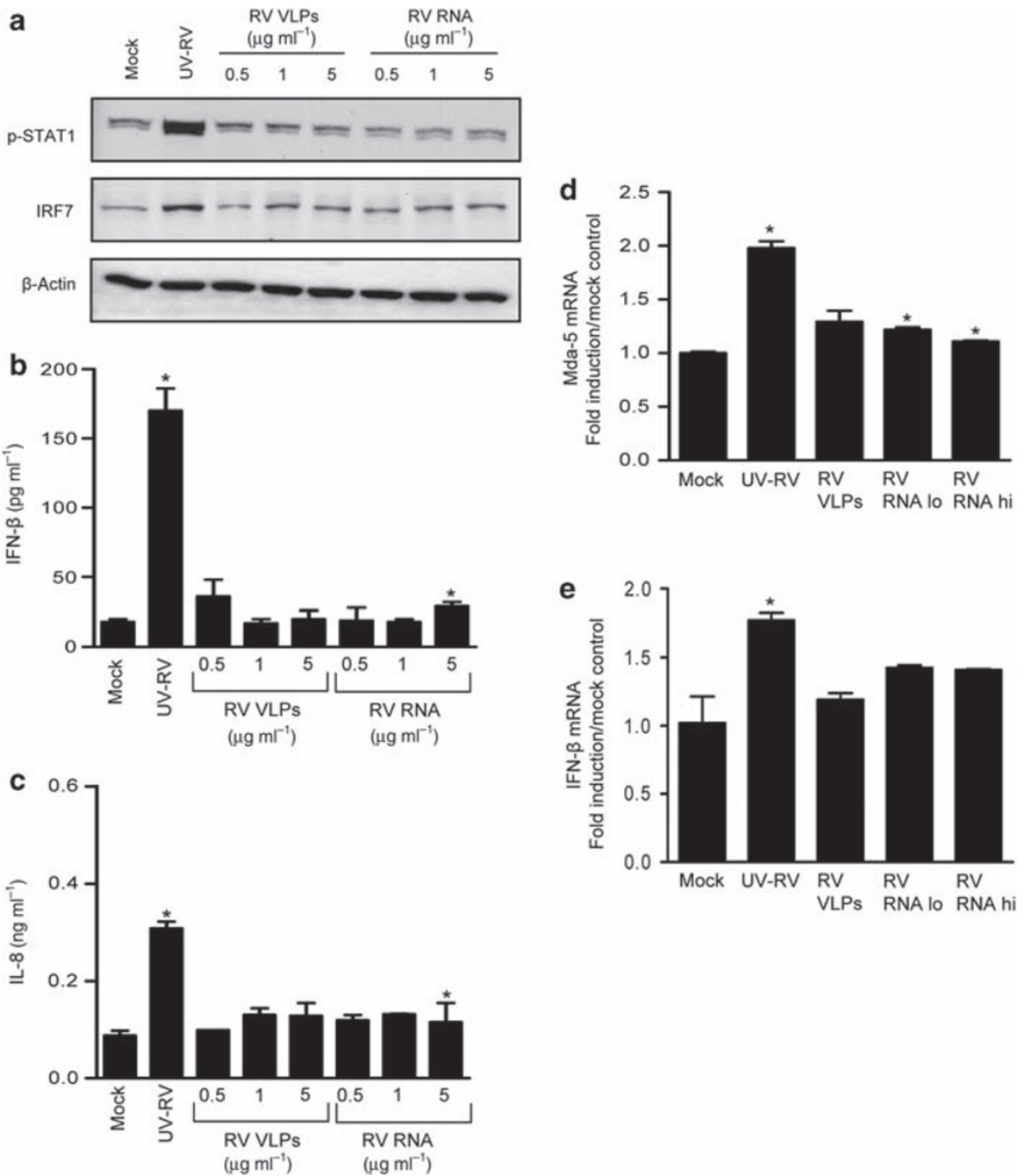

Figure 6 Anti-viral protein expression showed in epithelia treated with ultraviolet (UV)-rotavirus (RV), RV virus-like particles (VLPs), and RV RNA. Confluent intestinal epithelia (HT29) were grown in six-well plates and treated with UV-RV (MOI 0.5-1), RV RNA (0.5-5 $\mu$ g ml-1 ${ }^{-1}$, and VLPs $\left(0.5-5 \mathrm{~g} \mathrm{ml}^{-1}\right)$. Control samples received equivalent amounts of trypsin diluted in serum-free medium (SFM) (mock). Cell lysates and supernatants were collected at 24 h.p.i. Western blot analyses were used to detect anti-viral gene expression in cell lysates (a). Enzyme-linked immunosorbent assay (ELISA) assays were performed to measure interferon (IFN)- $\beta$ (b) or interleukin (IL)-8 (c) secretion in supernatants at $24 \mathrm{~h}$.p.i. Quantitative reverse transcriptase-PCR (qRT-PCR) analyses were used to confirm mRNA synthesis of select anti-viral genes from microarray experiments (see Table 3 ). Data in (a) are results of a single experiment and representative of three separate experiments that gave similar results. Data in (b-e) show the mean \pm s.e.m. of three parallel experiments. Statistically significant differences $P<0.05$ are denoted as starred values $\left(^{*}\right)$.

and/or their components might not only be useful for eliciting adaptive immune responses to protect against future infection, but may also help an infected host overcome the microbial innate immune suppression that can hinder pathogen clearance.

\section{METHODS}

Reagents. Rabbit anti-RV sera to purified rhesus RV was prepared as described previously. ${ }^{33}$ Mab to VP6, clone 6E7, was previously described. ${ }^{34}$ Antibodies to total and phosphorylated STAT1, IRF3, and PKR were obtained from Cell Signaling Technology (Beverly, MA). Total IRF7 antibodies were purchased from Santa Cruz Biotechnology (Santa Cruz, CA). Antibodies to human IFN alpha and beta (antiIFN $\alpha / \beta$ ) were obtained from the National Institute of Allergy and Infectious Diseases Reference Reagent Laboratory through ATCC
(Manassas, VA). Anti- $\beta$-actin and psoralen AMT (4'-aminomethyl4,5', 8-trimethylpsoralen) were purchased from Sigma-Aldrich (St Louis, MO). The enzyme-linked immunosorbent assay kit used to assay IFN- $\beta$ was obtained from PBL Biomedical Laboratories (Piscataway, NJ). IL-8 was assayed through R\&D systems Duoset IL-8 reagents (Minneapolis, MN).

Cell culture. Human IECs (HT29), herein referred to as "IEC", were cultured as previously described on both standard tissue-culture plastics or collagen-coated permeable supports to result in polarized IEC. ${ }^{35}$

Preparation of RV and components. Rhesus RV was propagated in MA104 cells as previously described. ${ }^{36}$ Virus was prepared in bulk, aliquoted, and stored at $-80^{\circ} \mathrm{C}$ until use. To prepare UV-inactivated $\mathrm{RV}$ (UV-RV), aliquots of rhesus RV were pretreated with $40 \mu \mathrm{g} \mathrm{ml}^{-1}$ 
Table 2 Genes induced by UV-RV, RV VLPs, and RV RNA relative to mock

\begin{tabular}{lcc}
\hline & \# genes & Avg. induction \\
\hline UV-RV & 401 & 1.5 \\
RV VLPS & 64 & 1.4 \\
RV RNA lo & 72 & 1.4 \\
RV RNA hi & 97 & 1.5 \\
\hline
\end{tabular}

Abbreviations: RV, rotavirus; SFM, serum-free medium; UV, ultraviolet; VLPS, virus-like particles.

Cellular gene transcription induced by UV-RV, RV VLP, and RV RNA. Intestinal epithelial (HT29) cell monolayers were stimulated for 24h with UV-RV (MOI 0.5), RV VLPs $\left(2.5 \mu \mathrm{g} \mathrm{ml}^{-1}\right)$, and RV RNA at low $\left(0.1 \mu \mathrm{g} \mathrm{ml}^{-1}\right)$ and high $\left(1 \mu \mathrm{g} \mathrm{ml}^{-1}\right)$ concentrations (denoted as RV RNA "Io" and "hi," respectively). Mock-treated samples received equivalent amounts of trypsin in SFM. Experiments were performed in biological duplicates. Lysates were collected in TRIzol to assess mRNA expression through microarray as described in text. The number of genes induced by UV-RV, RV VLPs, and RV RNA with >1.3-fold change is shown.

psoralen AMT and then irradiated by long-wave UV-light $(365 \mathrm{~nm})$ for $2 \mathrm{~h}$ as previously described. ${ }^{29}$ RV VLPs were isolated from Sf9 cells that had been infected with baculovirus recombinants expressing complementary DNAs encoding RV Rf VP2, and SA11 VP4, VP6, and VP7 as previously described. ${ }^{37} \mathrm{RV}$ RNA was extracted from purified virions as previously described. ${ }^{38,39}$

Cell treatment with RV and its components. Before infection, RV, UVRV, RV VLPs, and RV RNA were diluted in serum-free medium (SFM) and incubated with $10 \mu \mathrm{g} \mathrm{ml}^{-1}$ trypsin (Mediatech, Manassas, VA), except wherein indicated otherwise, for $30 \mathrm{~min}$ in a $37^{\circ} \mathrm{C}$ water bath. Control samples were treated with an equivalent amount of trypsin diluted in SFM (mock) or SFM alone. When indicated, neutralizing antibodies to human IFN alpha and beta (anti-IFN $\alpha / \beta)(1: 100)$ were added to some preparations of trypsinized RV before infection. IEC, HT29 cells, were grown to $90-100 \%$ confluence in six-well plates or collagen-coated permeable supports, washed several times with SFM, and inoculated with virus, viral components, or mock controls for $1 \mathrm{~h}$ at $37^{\circ} \mathrm{C} / 5 \% \mathrm{C}_{2}$ to allow for adsorption. After adsorption, cells were washed again several times with SFM and then incubated with $2 \mu \mathrm{g} \mathrm{ml}^{-1}$ trypsin in SFM for $0-48$ h.p.i. Cells stimulated with RV in the presence of anti-IFN $\alpha / \beta$ were treated with $2 \mu \mathrm{g} \mathrm{ml}^{-1}$ trypsin in SFM plus the same concentration of type 1 IFN antibodies used during viral adsorption. For experiments comparing RV VLPs and RNA, longer cell stimulation was required to allow enough time for RNA to enter cells; thus, after adsorption these cells were not washed with SFM and instead components were retained in the presence of $2 \mu \mathrm{g} \mathrm{ml}^{-1}$ trypsin for up to $24 \mathrm{~h}$. At the indicated time points, supernatants were collected and stored at $-20^{\circ} \mathrm{C}$ for IL-8/IFN- $\beta$ enzyme-linked immunosorbent assay. Cells were washed several times with phosphate-buffered saline (PBS) and resuspended in radioimmunoprecipitation assay II buffer (RIPA II) (20 mm Tris-HCl, $2.5 \mathrm{~mm}$ EDTA, $1 \%$ Triton X-100, $10 \%$ glycerol, $1 \%$ deoxycholate, $0.1 \%$ SDS, $50 \mathrm{~mm} \mathrm{NaF}$, $10 \mathrm{~mm} \mathrm{Na}_{2} \mathrm{P}_{2} \mathrm{O}_{7}$, and $2 \mathrm{~mm} \mathrm{NaV0}_{4}$ plus protease inhibitor cocktail) for western blotting or TRIzol for RNA isolation and subsequent qRT-PCR/ microarray analysis.

\section{Immunoblotting}

At various time points (0-48 h.p.i.), cells were rinsed several times in PBS, lysed in RIPA II buffer, and cleared by centrifugation (10 min at $15,000 \times g, 4^{\circ} \mathrm{C}$ ). Total protein concentrations were estimated by Bio-Rad Protein Assay (Hercules, CA). Cell lysates were assayed for anti-viral markers (IRF3, IRF7, STAT1, and PKR) by $12 \%$ sodium dodecyl sulfate-polyacrylamide gel electrophoresis immunoblotting and membranes were stripped and probed for $\beta$-actin as a loading control. Immunoblots were visualized with the ECL system (GE Healthcare, Piscataway, NJ).

\section{Enzyme-linked immunosorbent assay}

Supernatants were collected at different time points (0-48h.p.i.) and stored at $-20^{\circ} \mathrm{C}$ until use. Human IL- 8 secretion in the supernatants was measured by the Duoset kit from R\&D systems. The human IFN- $\beta$ enzyme immunoassay kit from PBL Biomedical Laboratories was used for the quantification of IFN- $\beta$ in the supernatants, according to the manufacturer's instructions.

\section{Microarray analyses}

Cells were exposed to RV or the indicated component for $24 \mathrm{~h}$, washed several times with PBS, resuspended in TRIzol, subjected to DNase I digestion, and purified for RNA by using a commercially available RNeasy Minikit from Qiagen (Valencia, CA). RNA concentration was measured using spectrophotometry and quality was assessed by Agilent BioAnalyzer analysis (Santa Clara, CA). Microarray analyses were performed at the Emory Biomarker Microarray Core, in which qualified RNA samples were reverse-transcribed, amplified, labeled, and used to probe human HT-12 chips purchased from Affymetrix (Santa Clara, CA). Briefly, samples were assayed using a Molecular Devices Gene Pix (4100A) (Silicon Valley, CA) and raw fluorescence readings were processed by an algorithm designed to reduce spurious readouts of gene activation. Microarray data were quantile normalized using freely available scripts written in R (http://R-project.org). Significantly altered genes were identified using significance of analysis of microarray analyses and assessed by hierarchal clustering and principle component analysis using Spotfire Decision Site for Functional Genomics software (TIBCO, Somerville, MA) to determine relatedness of gene expression patterns resulting from cell stimulation by RV or the indicated component.

\section{qRT-PCR}

At the indicated time points, total RNA was extracted from cells using TRIzol reagent and reverse-transcribed using a commercial kit (TaqMan Reverse Transcription kit; PerkinElmer, Boston, MA) according to the manufacturer's directions. The RT complementary DNA reaction products were subjected to quantitative real-time quantitative PCR (SYBR Green PCR Core kit; PerkinElmer) with primers for IFN- $\beta$ (sense $5^{\prime}$-CTCTCCTGTTGTGCTTCTCC-3', antisense 5'-GTCAAAGTTCATCCTGTCCTTG-3'), melanoma differentiationassociated gene 5 (sense $5^{\prime}$-TCAGCCAAATCTGGAGAAGG-3', antisense $5^{\prime}$-CTTCATCTGAATCACTTCCC-3'), STAT1 (sense 5'-GTTAGACAAACAGAAAGAGC-3', antisense 5' -TCTGTTGTGC AAGGTTTTGC-3'), oligoadenylate synthetase-2 (sense 5' -CAAC AAATGCTTCCTAGAGC-3' , antisense $5^{\prime}$-ACGAGATCGGCAT CAGAGCC-3') (Invitrogen, Carlsbad, CA), and 18S ribosomal RNA (sense 5' -CGGCTACCACATCCAAGGAA-3', antisense 5' -GCTGG AATTACCGCGGCT-3') (PerkinElmer) as previously described. ${ }^{40}$ Expression level of anti-viral genes was normalized to $18 \mathrm{~S}$ ribosomal RNA levels of the same sample. Fold difference was the ratio of the normalized value of each sample to that of uninfected control cells. All PCR samples were performed in triplicate.

\section{Confocal microscopy}

IEC were grown to confluence on collagen-coated permeable supports, apically treated with the indicated stimuli, and fixed in $10 \%$ formalin for $15 \mathrm{~min}$. Cells were washed $3 \times$ in PBS, permeabilized in $0.5 \%$ Triton $\mathrm{X}-100 / \mathrm{PBS}$ for $10 \mathrm{~min}$ at RT, and blocked overnight in $3 \%$ bovine serum albumin in PBS $\left(4^{\circ} \mathrm{C}\right)$. Cells were incubated for $1 \mathrm{~h}$ with polyclonal rabbit anti-rhesus RV $(1: 10,000)$ or monoclonal mouse anti-VP6 (1:100) in blocking buffer, washed with PBS $3 \times$, and probed with anti-rabbit and anti-mouse fluorescein isothiocyanate secondary antibody in PBS (1:50) (Jackson ImmunoResearch Laboratories, West Grove, PA), respectively, for $1 \mathrm{~h}$ at RT. Alexa-conjugated phalloidin stain (Alexa Fluor 633; Invitrogen) was used as a counterstain for F-actin and was included in the secondary antibody preparation at a dilution of 1:500. After staining with secondary antibody and 
Table 3 Fold change induction of anti-viral markers relative to mock

\begin{tabular}{lcccc}
\hline & UV-RV & RV VLPs & RV RNA lo & RV RNA hi \\
\hline Mda-5 & $1.8 \pm 0.18$ & $1.4 \pm 0.21$ & $1.2 \pm 0.03$ & $1.4 \pm 0.00$ \\
IFN- $\beta$ & $6.4 \pm 0.12$ & $1.2 \pm 0.06$ & $1.1 \pm 0.02$ & $1.3 \pm 0.21$ \\
MHC I & $1.7 \pm 0.14$ & $1.2 \pm 0.02$ & $1.1 \pm 0.03$ & $1.3 \pm 0.12$ \\
\hline
\end{tabular}

Abbreviations: IFN, interferon; Mda-5, melanoma differentiation-associated gene 5; MHC, major histocompatibility complex; qRT-PCR, quantitative reverse transcriptase-PCR; RV, rotavirus; UV, ultraviolet; VLP, virus-like particle.

Antiviral gene expression in cells treated with UV-RV, RV VLPs, and RV RNA. Cells were stimulated with UV-RV, RV VLPs, RV RNA, and mock treatments as described in Table 2. After 24h, lysates were collected in TRIzol to assess global transcription of genes (depicted in Table 2), including anti-viral markers of interest as indicated above. mRNA expression of select anti-viral genes was confirmed through qRT-PCR analysis (Figures $6 \mathbf{d}$ and $\mathbf{e}$ ). Fold induction values greater than s.d. calculations reflect statistically significant differences relative to mock $(P<0.05)$.

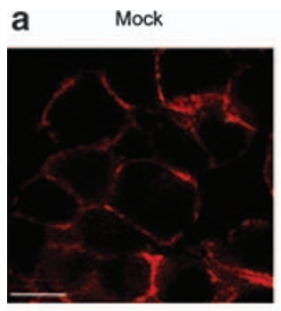

b

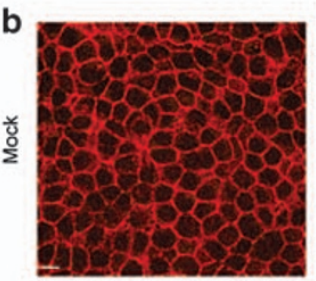

RV
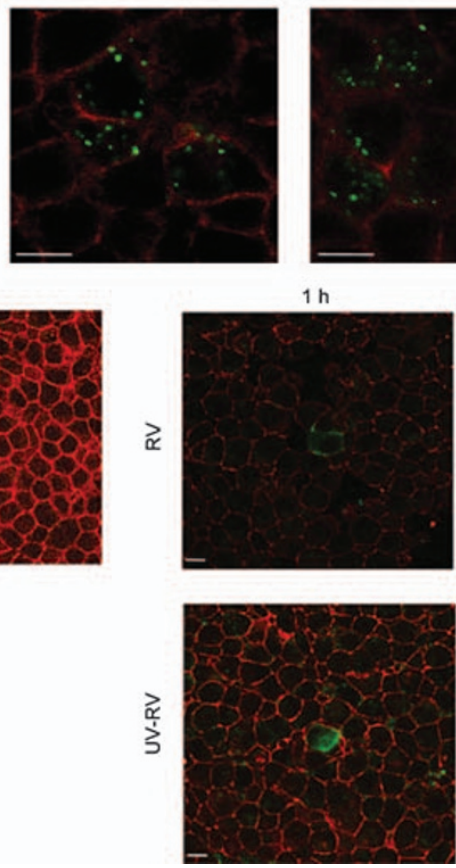

UV-RV
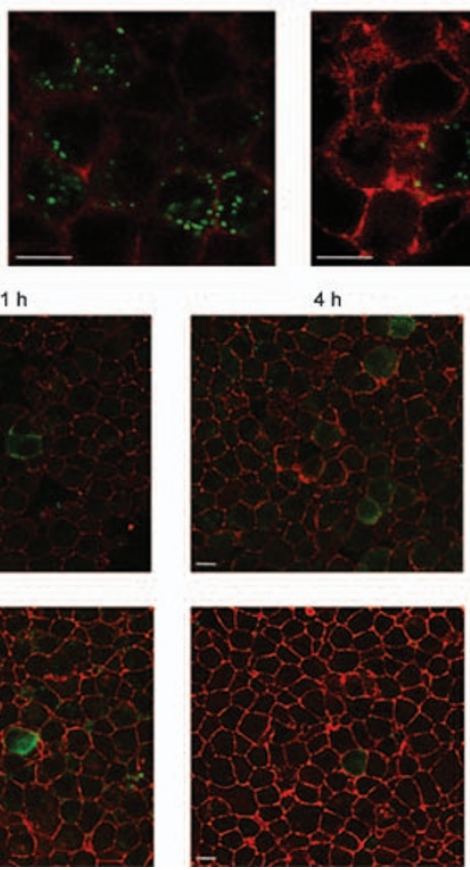

Figure 7 Rotavirus (RV), ultraviolet (UV)-RV, and virus-like particle (VLP) cell entry during early stages of infection. Intestinal epithelial monolayers (HT29) were grown on collagen-coated permeable supports and treated apically with RV and UV-RV (MOI 10), and an amount of VLPs roughly equivalent to the estimated protein concentration in the RV preparation. Control samples were apically treated with an equivalent amount of trypsin in serum-free medium (SFM) (mock) for 4 h.p.i. At 1 and 4 h.p.i., cells were fixed, stained, and examined through confocal fluorescence microscopy for presence of rotaviral proteins (green) and F-actin (red) in the sub-apical region of the cells ( $3 \mu \mathrm{m}$ below the apical surface). Sub-apical images of cells stimulated with RV, UV-RV, and RV VLPs at 1 h.p.i., magnification $\times 60$ (a). Sub-apical images of cells treated with RV and UV-RV at 1 and 4 h.p.i., magnification $\times 40$ (b). Data in $(\mathbf{a}$ and $\mathbf{b})$ are results of a single experiment and representative of three separate experiments. Scale reflects distance of $10 \mu \mathrm{m}$.

phalloidin, cells were washed $3 \times$ in PBS and mounted on slides with fluorescent anti-fade medium (VectaShield, Burlingame, CA). Stained cell monolayers were examined using a Zeiss LSM510 laser scanning confocal microscope (Zeiss Microimaging, Thornwood, NY) coupled to a Zeiss $100 \mathrm{M}$ axiovert and $\times 63$ or $\times 100$ Pan-Apochromat oil lenses. Fluorescent dyes were imaged sequentially in frame-interlace mode to eliminate cross-talk between channels. Images shown are representative of at least three experiments, with multiple images taken per slide.

SUPPLEMENTARY MATERIAL is linked to the online version of the paper at http://www.nature.com/mi

\section{ACKNOWLEDGMENTS}

We thank Drs Rheinallt Jones and Huxia Wu (Emory University, Atlanta, GA) for their advice and technical assistance. This work was supported by NIH grant Al083420 to A.T.G., Al080656 to M.K.E., a Digestive Disease Research and Development Center (DDRDC) grant DK06439, and a Digestive Diseases Research Core Center (DDRCC) grant DK66338.
The findings and conclusions in this report are those of the authors and do not necessarily represent the views of the CDC.

\section{DISCLOSURE}

The authors declared no conflict of interest.

C 2010 Society for Mucosal Immunology

\section{REFERENCES}

1. Glass, R.I. et al. Rotavirus vaccines: current prospects and future challenges. Lancet 368, 323-332 (2006).

2. Parashar, U.D., Hummelman, E.G., Bresee, J.S., Miller, M.A. \& Glass, R.I. Global illness and deaths caused by rotavirus disease in children. Emerg. Infect. Dis. 9, 565-572 (2003).

3. Anderson, E.J. \& Weber, S.G. Rotavirus infection in adults. Lancet Infect. Dis. 4, 91-99 (2004).

4. Greenberg, H.B. \& Estes, M.K. Rotaviruses: from pathogenesis to vaccination. Gastroenterology 136, 1939-1951 (2009). 
5. Jayaram, H., Estes, M.K. \& Prasad, B.V. Emerging themes in rotavirus cell entry, genome organization, transcription and replication. Virus Res. 101, 67-81 (2004).

6. Riepenhoff-Talty, M., Dharakul, T., Kowalski, E., Michalak, S. \& Ogra, P.L. Persistent rotavirus infection in mice with severe combined immunodeficiency. J. Virol. 61, 3345-3348 (1987).

7. Franco, M.A. \& Greenberg, H.B. Immunity to rotavirus in T cell deficient mice. Virology 238, 169-179 (1997).

8. Eiden, J., Lederman, H.M., Vonderfecht, S. \& Yolken, R. T-cell-deficient mice display normal recovery from experimental rotavirus infection. J. Virol. 57, 706-708 (1986).

9. Sheth, R. et al. Rotavirus stimulates IL-8 secretion from cultured epithelial cells. Virology 221, 251-259 (1996).

10. Casola, A. et al. Rotavirus infection of cultured intestinal epithelial cells induces secretion of CXC and CC chemokines. Gastroenterology 114, 947-955 (1998).

11. Rollo, E.E. et al. The epithelial cell response to rotavirus infection. J. Immunol. 163, 4442-4452 (1999).

12. Cuadras, M.A., Feigelstock, D.A., An, S. \& Greenberg, H.B. Gene expression pattern in Caco-2 cells following rotavirus infection. J. Virol. 76, 4467-4482 (2002).

13. Bass, D.M. Interferon gamma and interleukin 1 , but not interferon alfa, inhibit rotavirus entry into human intestinal cell lines. Gastroenterology 113, 81-89 (1997).

14. Lecce, J.G., Cummins, J.M. \& Richards, A.B. Treatment of rotavirus infection in neonate and weanling pigs using natural human interferon alpha. Mol. Biother. 2, 211-216 (1990).

15. Schwers, A., Vanden Broecke, C., Maenhoudt, M., Beduin, J.M., Werenne, J. \& Pastoret, P.P. Experimental rotavirus diarrhoea in colostrum-deprived newborn calves: assay of treatment by administration of bacterially produced human interferon (Hu-IFN alpha 2). Ann. Rech. Vet. 16, 213-218 (1985).

16. Wang, Y. et al. Rotavirus infection alters peripheral T-cell homeostasis in children with acute diarrhea. J. Virol. 81, 3904-3912 (2007).

17. Barro, M. \& Patton, J.T. Rotavirus nonstructural protein 1 subverts innate immune response by inducing degradation of IFN regulatory factor 3. Proc. Natl. Acad. Sci. USA 102, 4114-4119 (2005).

18. Barro, M. \& Patton, J.T. Rotavirus NSP1 inhibits expression of type I interferon by antagonizing the function of interferon regulatory factors IRF3, IRF5, and IRF7. J. Virol. 81, 4473-4481 (2007).

19. Feng, N. et al. Role of interferon in homologous and heterologous rotavirus infection in the intestines and extraintestinal organs of suckling mice. J. Virol. 82, 7578-7590 (2008).

20. Vancott, J.L., McNeal, M.M., Choi, A.H. \& Ward, R.L. The role of interferons in rotavirus infections and protection. J. Interferon Cytokine Res. 23, 163-170 (2003).

21. He, B. Viruses, endoplasmic reticulum stress, and interferon responses. Cell Death Differ. 13, 393-403 (2006).

22. Zeng, $H$. et al. Flagellin is the major proinflammatory determinant of enteropathogenic Salmonella. J. Immunol. 171, 3668-3674 (2003).
23. Gewirtz, A.T. Intestinal epithelial toll-like receptors: to protect. And serve? Curr. Pharm. Des. 9, 1-5 (2003).

24. Vijay-Kumar, M. et al. Protein kinase R mediates intestinal epithelial gene remodeling in response to double-stranded RNA and live rotavirus. J. Immunol. 174, 6322-6331 (2005).

25. Estes, M.K., Graham, D.Y. \& Mason, B.B. Proteolytic enhancement of rotavirus infectivity: molecular mechanisms. J. Virol. 39, 879-888 (1981).

26. Gewirtz, A.T., Siber, A.M., Madara, J.L. \& McCormick, B.A. Orchestration of neutrophil movement by intestinal epithelial cells in response to Salmonella typhimurium can be uncoupled from bacterial internalization. Infect. Immun. 67, 608-617 (1999).

27. Ciarlet, M., Crawford, S.E. \& Estes, M.K. Differential infection of polarized epithelial cell lines by sialic acid-dependent and sialic acid-independent rotavirus strains. J. Virol. 75, 11834-11850 (2001).

28. Gewirtz, A.T., Navas, T.A., Lyons, S., Godowski, P.J. \& Madara, J.L. Cutting edge: bacterial flagellin activates basolaterally expressed TLR5 to induce epithelial proinflammatory gene expression. J. Immunol. 167, 1882-1885 (2001).

29. Groene, W.S. \& Shaw, R.D. Psoralen preparation of antigenically intact noninfectious rotavirus particles. J. Virol. Methods 38, 93-102 (1992).

30. Shaw, R.D., Hempson, S.J. \& Mackow, E.R. Rotavirus diarrhea is caused by nonreplicating viral particles. J. Virol. 69, 5946-5950 (1995).

31. Jiang, B. et al. Heterotypic protection from rotavirus infection in mice vaccinated with virus-like particles. Vaccine 17, 1005-1013 (1999).

32. Vijay-Kumar, M. et al. Flagellin treatment protects against chemicals, bacteria, viruses, and radiation. J. Immunol. 180, 8280-8285 (2008).

33. Gerna, G. et al. Isolation and characterization of two distinct human rotavirus strains with G6 specificity. J. Clin. Microbiol. 30, 9-16 (1992).

34. Blutt, S.E. et al. Rotavirus antigenemia in children is associated with viremia. PLoS Med. 4, e121 (2007).

35. Nash, S., Parkos, C., Nusrat, A., Delp, C. \& Madara, J.L. In vitro model of intestinal crypt abscess. A novel neutrophil-derived secretagogue activity. J. Clin. Invest. 87, 1474-1477 (1991).

36. Shaw, R.D., Stoner-Ma, D.L., Estes, M.K. \& Greenberg, H.B. Specific enzyme-linked immunoassay for rotavirus serotypes 1 and 3. J. Clin. Microbiol. 22, 286-291 (1985).

37. Crawford, S.E., Labbe, M., Cohen, J., Burroughs, M.H., Zhou, Y.J. \& Estes, M.K. Characterization of virus-like particles produced by the expression of rotavirus capsid proteins in insect cells. J. Virol. 68, 5945-5952 (1994).

38. Boom, R., Sol, C.J., Salimans, M.M., Jansen, C.L., Wertheim-van Dillen, P.M. \& van der Noordaa, J. Rapid and simple method for purification of nucleic acids. J. Clin. Microbiol. 28, 495-503 (1990).

39. Gentsch, J.R. et al. Identification of group A rotavirus gene 4 types by polymerase chain reaction. J. Clin. Microbiol. 30, 1365-1373 (1992).

40. Evans, C.O. et al. Novel patterns of gene expression in pituitary adenomas identified by complementary deoxyribonucleic acid microarrays and quantitative reverse transcription-polymerase chain reaction. J. Clin. Endocrinol. Metab. 86, 3097-3107 (2001). 